\title{
TU/e EmonONEN

\section{Two interacting atoms in an optical lattice site with anharmonic terms}

Citation for published version (APA):

Mentink, J. H., \& Kokkelmans, S. J. J. M. F. (2009). Two interacting atoms in an optical lattice site with anharmonic terms. Physical Review A : Atomic, Molecular and Optical Physics, 79(3), 032709-9. [032709]. https://doi.org/10.1103/PhysRevA.79.032709

DOI:

10.1103/PhysRevA.79.032709

Document status and date:

Published: 01/01/2009

\section{Document Version:}

Publisher's PDF, also known as Version of Record (includes final page, issue and volume numbers)

\section{Please check the document version of this publication:}

- A submitted manuscript is the version of the article upon submission and before peer-review. There can be important differences between the submitted version and the official published version of record. People interested in the research are advised to contact the author for the final version of the publication, or visit the $\mathrm{DOI}$ to the publisher's website.

- The final author version and the galley proof are versions of the publication after peer review.

- The final published version features the final layout of the paper including the volume, issue and page numbers.

Link to publication

\section{General rights}

Copyright and moral rights for the publications made accessible in the public portal are retained by the authors and/or other copyright owners and it is a condition of accessing publications that users recognise and abide by the legal requirements associated with these rights.

- Users may download and print one copy of any publication from the public portal for the purpose of private study or research.

- You may not further distribute the material or use it for any profit-making activity or commercial gain

- You may freely distribute the URL identifying the publication in the public portal.

If the publication is distributed under the terms of Article 25fa of the Dutch Copyright Act, indicated by the "Taverne" license above, please follow below link for the End User Agreement:

www.tue.nl/taverne

Take down policy

If you believe that this document breaches copyright please contact us at:

openaccess@tue.nl

providing details and we will investigate your claim. 


\title{
Two interacting atoms in an optical lattice site with anharmonic terms
}

\author{
Johan Mentink* and Servaas Kokkelmans \\ Eindhoven University of Technology, P.O. Box 513, 5600 MB Eindhoven, The Netherlands. \\ (Received 29 June 2008; revised manuscript received 22 January 2009; published 19 March 2009)
}

\begin{abstract}
We propose an easy to use model for interacting atoms in an optical lattice. This model allows for the whole range of weakly to strongly interacting atoms, and it includes the coupling between relative and center-of-mass motions via anharmonic lattice terms. We apply this model to a high-precision spin-dynamics experiment, and we discuss the corrections due to atomic interactions and the anharmonic coupling. Under suitable experimental conditions, energy can be transferred between the relative and center-of-mass motions, and this allows for creation of Feshbach molecules in excited lattice bands.
\end{abstract}

DOI: 10.1103/PhysRevA.79.032709

PACS number(s): 34.50.-s, 37.10.Jk, 03.75.Lm, 34.50.Rk

\section{INTRODUCTION}

Optical lattices form a suitable environment for highprecision experiments with interacting atoms. Two atoms can be isolated from other atoms by placing them on a single lattice site, and many sites can be filled simultaneously. While the lattice parameters such as depth and geometry can be adjusted via the laser field, the interactions can be tuned using a Feshbach resonance by applying an external magnetic field. Feshbach molecules in a lattice, created by sweeping the magnetic field over resonance, can be transferred into deeper vibrational bound states, for instance, by applying stimulated Raman adiabatic passage [1].

Precise values for relative interaction strengths of rubidium atoms were derived by studying coherent collisional spin dynamics in an optical lattice [2,3]. These highprecision measurements provide challenges for theoretical coupled-channel models based on current state-of-the-art interaction potentials [4]. One may wonder, for instance, at what level of precision it is possible to calculate interaction properties before the Born-Oppenheimer approximation, which is the underlying foundation for the potentials, breaks down.

Before conclusions can be drawn on the limitations of two-body interaction models, one has to make sure that the correct comparison is made between theoretical quantities of such a calculation, and the measurements that depend on these interactions. For instance, one cannot always put the resulting scattering lengths of a two-body collision, defined in the limit of zero collision energy, as the on-site interaction in a Hubbard model. The divergence of the scattering length on resonance will give rise to physically unrealistic large energy shifts. A resonant interaction takes the two-body interaction in the unitarity limit, where the solution of scattering wave functions are shifted over $\pi / 2$ compared to noninteracting atoms, and one would rather use expressions based on the (energy) dependent scattering phase shift. This argument also applies for high-precision experiments on nonresonant systems since small energy-dependent corrections already can be of importance. Also, the relative and center-of-

\footnotetext{
*Present address: Institute for Molecules and Materials, Radboud University, Nijmegen, The Netherlands
}

mass motions of two interacting atoms, which can become coupled due to different atomic species $[5,6]$ and anharmonic terms in the lattice potential [6], can give rise to shifts in the on-site interaction.

The model we put forward in this paper is conceptionally simple and easy to use. It is based on first-order perturbation theory starting from an existing solution of two interacting atoms in a harmonic potential. We show that the model is valid for moderately deep lattices, i.e., where tunneling to neighboring sites is negligible. In this way, we are able to make a proper comparison between the high-precision measurements by Widera et al. [2] and accurate rubidium scattering models. We demonstrate the importance of energy dependence in the scattering phase shift and of anharmonic corrections for this experiment, and also show how experiments using a Feshbach resonance can produce molecules of a mixed relative and center-of-mass motion nature.

This paper is outlined as follows: In Sec. II we give a description of our model. Then we apply it to a spindynamics experiment in a lattice in Sec. III. In Sec. IV, we discuss the nature of the molecules that have a mixed relative and center-of-mass motion, predicted by our model. We end with Sec. V.

\section{MODEL}

In the ultracold regime only $s$-wave interactions are allowed, and we conveniently model the interaction with a pseudopotential [7]

$$
V_{\mathrm{int}}(r)=\frac{4 \pi \hbar^{2}}{m} a \delta^{(3)}(r) \frac{\partial}{\partial r} r .
$$

Here $r$ is the distance between two colliding atoms, and $a$ is the $s$-wave scattering length [8]. The use of this zero-range pseudopotential is allowed since $R_{\mathrm{vdW}} \ll d$ [9], with

$$
R_{\mathrm{vdW}}=\frac{1}{2}\left(\frac{m C_{6}}{\hbar^{2}}\right)^{1 / 4}
$$

as the van der Waals length defining the range of the real interaction potentials [10] with $C_{6}$ as the leading dispersion coefficient of the tail of the potential, and $d=\pi / k_{L}$ as the lattice spacing, where $k_{L}$ is the wave number corresponding to the laser frequency. 
The total Hamiltonian for a pair of interacting atoms in an optical lattice is described by

$$
\begin{aligned}
H= & -\frac{\hbar^{2}}{2 m}\left(\frac{1}{2} \nabla_{\mathbf{R}}^{2}+2 \nabla_{\mathbf{r}}^{2}\right)+V_{\text {lat }}\left(\mathbf{R}+\frac{\mathbf{r}}{2}\right)+V_{\text {lat }}\left(\mathbf{R}-\frac{\mathbf{r}}{2}\right) \\
& +V_{\text {int }}(r) .
\end{aligned}
$$

Here

$$
V_{\text {lat }}(\mathbf{x})=\sum_{j=1}^{3} V_{j}\left(x_{j}\right), \quad V_{j}\left(x_{j}\right)=V_{0} \sin ^{2}\left(k_{L} x_{j}\right)
$$

is the optical lattice potential with depth $V_{0}$ and $\mathbf{x}$ $=\left(x_{1}, x_{2}, x_{3}\right)$ is decomposed along the lattice axes. $\mathbf{R}$ and $\mathbf{r}$ denote the center-of-mass (CM) and relative positions of the atoms.

This Hamiltonian gives rise to nonseparable solutions in these two coordinates. Moreover, $V_{\text {int }}$ breaks the translational invariance in the relative coordinate while the periodicity in the center of mass is still intact. This periodicity ensures that, when $\psi(\mathbf{r}, \mathbf{R})$ is a solution, $\psi(\mathbf{r}, \mathbf{R}+\mathbf{D})$ is a solution as well. We can formulate this in terms of the Bloch theorem

$$
\psi_{\mathbf{Q}}(\mathbf{r}, \mathbf{R}+\mathbf{D})=e^{\mathrm{i} \mathbf{Q} \cdot \mathbf{D}} \psi_{\mathbf{Q}}(\mathbf{r}, \mathbf{R})
$$

with $\mathbf{Q}$ as the quasimomentum associated to the center-ofmass motion of this two-particle wave function [11].

\section{A. Harmonic approximation}

The restriction to a pair of interacting atoms is an approximation but becomes exact for deep lattices (for example, in the Mott-insulator regime) with two trapped atoms per lattice site. This regime will be our starting point. Then, separation of the CM and relative motions is possible when each site is treated as a harmonic oscillator (HO) with frequency $\omega$ $=\sqrt{2 V_{0} k_{L}^{2} / m}$. The lattice potential for two particles can then be written as

$$
V_{\text {lat }}\left(\mathbf{R}+\frac{\mathbf{r}}{2}\right)+V_{\text {lat }}\left(\mathbf{R}-\frac{\mathbf{r}}{2}\right) \approx \frac{1}{2} m \omega^{2}\left(2 \mathbf{R}^{2}+\frac{1}{2} \mathbf{r}^{2}\right) .
$$

An exact solution for two interacting atoms in a HO trap can be derived for the relative motion, as was first shown by Busch et al. [12]. When we expand the angular part to the angular-momentum basis and we restrict ourselves to the case $l=0$ ( $s$ waves), then we are left with a one-dimensional (1D) Schrödinger equation for the relative radial coordinate,

$$
\left[-\frac{\hbar^{2}}{2 \mu} \nabla^{2}+\frac{1}{2} \mu \omega^{2} r^{2}+\frac{2 \pi \hbar^{2}}{\mu} a \delta(r) \frac{\partial}{\partial r} r\right] \psi(r)=e \psi(r),
$$

with $\mu=m / 2$ as the reduced mass, and $\delta(r)$ as the 1D delta function.

The contact potential can be interpreted as a boundary condition for the harmonic-oscillator equation, and solutions are given in terms of a relation between the scattering length and the (modified) harmonic-oscillator levels,

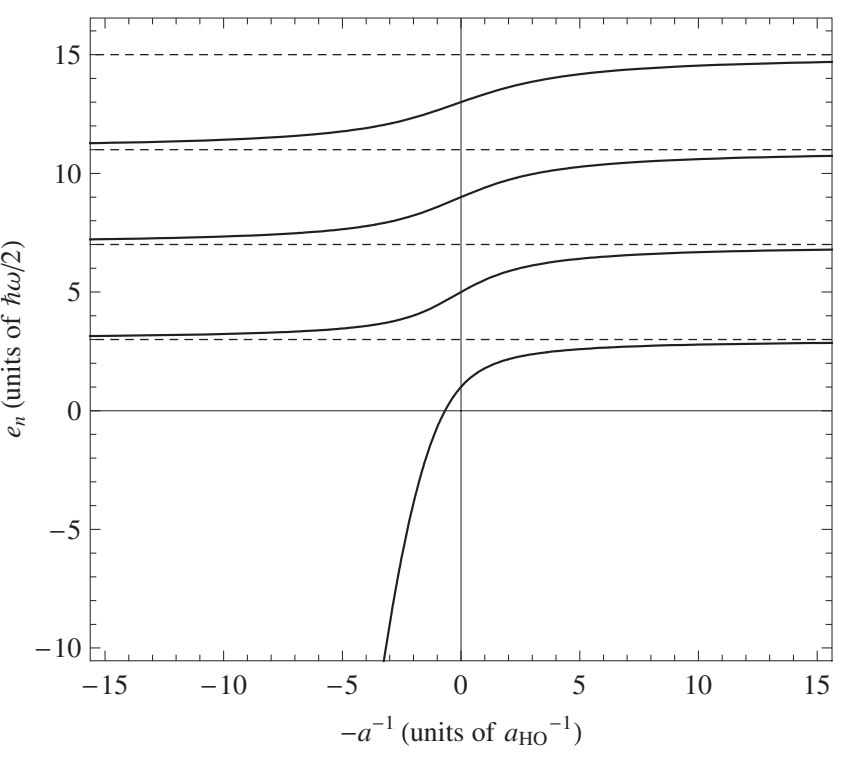

FIG. 1. Lowest branches of the energy spectrum $e_{n}(a)$ for two particles in a harmonic trap, with the interaction modeled by a contact potential. Relative energy is plotted as function of the reciprocal scattering length. The scattering length is scaled on $a_{\mathrm{HO}}$ $=\sqrt{2 \hbar /(m \omega)}$, the harmonic-oscillator length of the relative motion. The dashed lines denote the energy levels for vanishing interaction.

$$
\frac{1}{2\left(a / a_{\mathrm{HO}}\right)}=\frac{\Gamma\left(\frac{3}{4}-\frac{e}{2 \hbar \omega}\right)}{\Gamma\left(\frac{1}{4}-\frac{e}{2 \hbar \omega}\right)},
$$

with $a_{\mathrm{HO}}=\sqrt{2 \hbar /(m \omega)}$ as the harmonic-oscillator length of relative motion. The relation between $e(a)$ and $a$ is not unique, i.e., for each value of $a$, there are infinitely many different values $e(a)$ that satisfy Eq. (8) just as there are infinitely many different $\mathrm{HO}$ energy levels for the case without interaction. We label the different branches with index $n=1,3,5, \ldots$, and the corresponding eigenvalues as $e_{n}(a)$. Note that in general even values of $l$ correspond to odd values for $n$, and accordingly that all odd values of $l$ correspond to even values for $n$ (except $n=0$ ) [13].

Figure 1 shows the lowest few branches of the eigenvalue spectrum $e_{n}(a)$. The corresponding eigenfunctions are given by

$$
\phi_{n}(r)=A_{n} e^{-\left(r / a_{\mathrm{HO}}\right)^{2} / 2} U\left[\frac{3}{4}-\frac{e_{n}(a)}{2 \hbar \omega}, \frac{3}{2},\left(\frac{r}{a_{\mathrm{HO}}}\right)^{2}\right],
$$

where $U(b, c, z)$ is a confluent hypergeometric function of the second kind, and $A_{n}$ is a normalization constant.

Note that the ground state has a quite distinct behavior compared to the excited states in the regime where $a$ becomes smaller than $a_{\mathrm{HO}}: a_{\mathrm{HO}} / a>1$. This is due to the presence of a bound state in the interatomic potential, and the ground state will evolve into this bound state for $a<a_{\mathrm{HO}}$, with a corresponding binding energy of $e_{n}(a) /(\hbar \omega / 2)$ $=-\left(a_{\mathrm{HO}} / a\right)^{2}$. Accordingly, the spatial extension of the wave function is then no longer given by the harmonic-oscillator length $a_{\mathrm{HO}}$ but by the scattering length $a$, 


$$
\phi_{1}(r) \sim \frac{1}{r} e^{-r / a}
$$

We come back to the creation of these molecules in the presence of anharmonic contributions in Sec. IV. Figure 1 also shows that sweeping through a Feshbach resonance can give rise to a transfer of atoms from one spherically symmetric $\mathrm{HO}$ state to the next one, and therefore it is possible to increase or decrease the relative energy by $2 \hbar \omega$.

In order to model lattice effects beyond the harmonic approximation, we have to incorporate anharmonic terms as well as tunneling. These effects can be of significant importance even when the atoms are considered trapped in a single site $[3,6]$.

\section{B. Anharmonic contributions}

In this paper, we propose to handle anharmonic terms in a perturbative procedure, as we will discuss below. First we argue that for sufficiently deep lattices tunneling effects are small compared to anharmonic effects while anharmonic terms have a small but significant effect.

The significance of tunneling and anharmonic terms can be estimated using the complete lattice solution for the case without interaction. In the Appendix we show that this solution can then be conveniently expressed in terms of Mathieu functions.

Based on this exact solution we estimate the significance of tunneling and anharmonic terms for the lowest three bands. Tunneling induces a broadening of the energy levels of an individual well indicated as $W=E_{t}-E_{b}$ and gives rise to the band structure of a square lattice [11]. In addition, anharmonic terms shift a level compared to the corresponding $\mathrm{HO}$ level $E_{\mathrm{HO}}$ by an amount $\Delta E=E_{\mathrm{HO}}-\left(E_{t}+E_{b}\right) / 2$. Here $E_{t}$ and $E_{b}$ are the band top and band bottom energies, respectively.

The results of these estimates are shown in Fig. 2. It can be seen that, sufficiently deep in the Mott phase, in particular for lattice depths $V_{0} / E_{\text {rec }}$ as used in current experiments, the anharmonic terms dominate above tunneling: $W \ll|\Delta E|$. Also, the inset of Fig. 2 clearly illustrates that a level shift remains present even when tunneling vanishes. This remaining shift is thus caused by anharmonic terms and is typically of order $|\Delta E| / E_{\mathrm{HO}} \sim 5 \%$. Hence, we have the following set of inequalities for sufficiently deep lattices,

$$
W \ll|\Delta E| \ll E_{\mathrm{HO}} .
$$

We therefore expect that for arbitrary values of the scattering length we can successfully apply a perturbative approach. In this paper, we use the HO solution as zeroth order solution and include anharmonic terms as perturbation. In principle, we can also use the wave function including anharmonic corrections to compute the broadening of the levels from the overlap between these wave functions in neighboring lattice sites. However, for the purpose of our present results this is not necessary since they are already sufficiently accurate for optical lattices where we can neglect the effect of tunneling.

We write the zeroth order wave function as

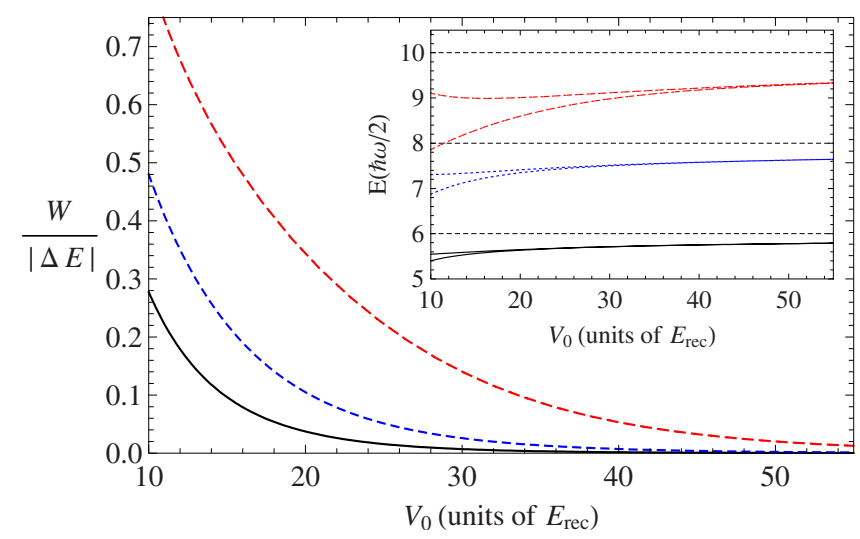

FIG. 2. (Color online) Estimation of tunneling and anharmonic effects based on exact lattice solutions for noninteracting particles. The ratio of bandwidth $W$ and level shift $\Delta E$ is shown as function of lattice depth $V_{0}$, given in units of recoil energy $E_{\mathrm{rec}}=\hbar^{2} k_{L}^{2} / 2 m$. The solid and dashed-dotted lines correspond to the lowest and secondbut-lowest symmetric bands, respectively. The dashed line represents the lowest antisymmetric band. The inset shows the total HO energies (dashed lines) and corresponding band tops and bottoms from which the main graph is computed.

$$
\psi_{S s}^{(0)}(\mathbf{R}, \mathbf{r})=\Phi_{S}(\mathbf{R}) \phi_{S}(\mathbf{r})
$$

Here $\Phi_{S}(\mathbf{R})$ and $\phi_{s}(\mathbf{r})$ are the exact HO solutions of the CM and relative motions, corresponding to states labeled with $S$ $=\left\{S_{1}, S_{2}, S_{3}\right\}$ and $s=\{n, l, m\}$, respectively. We use different quantum numbers for $\mathrm{CM}$ and relative motions to adapt optimally to both the cubic symmetry of the perturbation term [Eq. (13)] and the spherical symmetry of the interaction region. Therefore, $\mathrm{CM}$ is always described in Cartesian coordinates, with quantum numbers $S_{j}=0,1,2, \ldots$, for the different lattice axes. Relative motion is decomposed in spherical coordinates, with principal quantum number $n=1,2,3, \ldots$, and orbital quantum numbers $l$ and $m$.

As perturbation term we have

$$
\begin{aligned}
H^{\prime}(\mathbf{R}, \mathbf{r})= & V_{\text {lat }}\left(\mathbf{R}+\frac{\mathbf{r}}{2}\right)+V_{\text {lat }}\left(\mathbf{R}-\frac{\mathbf{r}}{2}\right) \\
& +-\frac{1}{2} m \omega^{2}\left(2 \mathbf{R}^{2}+\frac{1}{2} \mathbf{r}^{2}\right) .
\end{aligned}
$$

We define our perturbative solutions in accordance with standard perturbation theory,

$$
E_{S s}=E_{S s}^{(0)}+\left\langle\psi_{S s}^{(0)}\left|H^{\prime}\right| \psi_{S s}^{(0)}\right\rangle+\ldots,
$$

and

$$
\psi_{S s}=\psi_{S s}^{(0)}+\sum_{\substack{S^{\prime} \neq S \\ s^{\prime} \neq s}} \frac{\left\langle\psi_{S^{\prime} s^{\prime}}^{(0)}\left|H^{\prime}\right| \psi_{S s}^{(0)}\right\rangle}{E_{S s}^{(0)}-E_{S^{\prime} s^{\prime}}^{(0)}} \psi_{S^{\prime} s^{\prime}}^{(0)}+\ldots
$$

for the first-order corrected energies and wave functions, respectively. $E_{S s}^{(0)}=E_{S}+e_{s}$ is the sum of the $\mathrm{HO}$ energies corresponding to the $\mathrm{CM}$ and relative motions. The zeroth order relative wave function and energy depend parametrically on the scattering length according to the Busch model [12]: 
$\phi_{s}(\mathbf{r})=\phi_{s}(\mathbf{r} ; a)$ and $e_{s}=e_{s}(a)$. Hence the anharmonic correction becomes a function of scattering length as well. Note however that the perturbation term itself does not depend on the scattering length, illustrating that the interaction is modeled exactly.

The wave function $\psi_{S s}$ obtained from Eq. (15) only satisfies the boundary conditions for the relative motion. We can also satisfy the periodic CM boundary conditions [Eq. (5)] by constructing the lattice solution as

$$
\psi_{\mathbf{Q} S s}(\mathbf{R}, \mathbf{r})=\sum_{\mathbf{D}} e^{i \mathbf{Q} \cdot \mathbf{D}} \psi_{S S}(\mathbf{R}-\mathbf{D}, \mathbf{r}),
$$

with direct lattice vector $\mathbf{D}=\left(n_{1} d, n_{2} d, n_{3} d\right)$, integers $n_{i}$, and $\mathbf{Q}$ as the CM quasimomentum introduced in Eq. (5). This demonstrates that the wave function $\psi_{S s}$ obtained with our model is effectively a Wannier function of a pair of interacting atoms in an optical lattice.

Finally, we check the accuracy of our model by using again the exact lattice solution, which is available for the case without interatomic interactions. Regarding the energy, we compare the total energy $E_{S s}$ with $E_{\text {exact }}=\left(E_{t}+E_{b}\right) / 2$. In addition, we compare the first- and second-order corrections with the exact shift, and compare this with the contribution of tunneling. All calculations are performed at a lattice depth $V_{0}=25 E_{\text {rec }}$. For the total energy we find already with only the first-order correction,

$$
\left(E_{S s}-E_{\text {exact }}\right) / E_{\text {exact }}<0.7 \% .
$$

The first-order correction covers almost $90 \%$ of the exact shift,

$$
\left\langle\psi_{S S}^{(0)}\left|H^{\prime}\right| \psi_{S s}^{(0)}\right\rangle / \Delta E \approx 0.88 .
$$

The second-order correction is one order-of-magnitude smaller,

$$
\frac{1}{\Delta E} \sum_{S^{\prime} \neq S} \frac{\left|\left\langle\psi_{S^{\prime} s^{\prime}}^{(0)}\left|H^{\prime}\right| \psi_{S s}^{(0)}\right\rangle\right|^{2}}{E_{S s}^{(0)}-E_{S^{\prime} s^{\prime}}^{(0)}} \approx 0.09
$$

This is of the same order as the relative contribution of tunneling (see also Fig. 2),

$$
W / \Delta E \approx 0.02 \text {. }
$$

Therefore, the meaning of second-order terms only is limited. Naturally, the accuracy improves when lattice depth is increased. We also compared the results for the wave function and an example is given in Fig. 3 for the same value of the lattice depth. Shown are the first-order corrected wave function, together with the zeroth order HO solution and the Wannier function, being the exact solution for a single site (see Appendix). Anharmonic corrections make the trap less tight compared to the $\mathrm{HO}$ approximation, resulting in a small decrease in the probability for finding the particles near $r$ $=0$ around the origin, corresponding to a small increase in probability density in the barrier. The difference between the Wannier function and the HO wave function with anharmonic corrections is small compared to the difference between the latter and the unperturbed $\mathrm{HO}$ wave function. This shows that the method converges quickly.

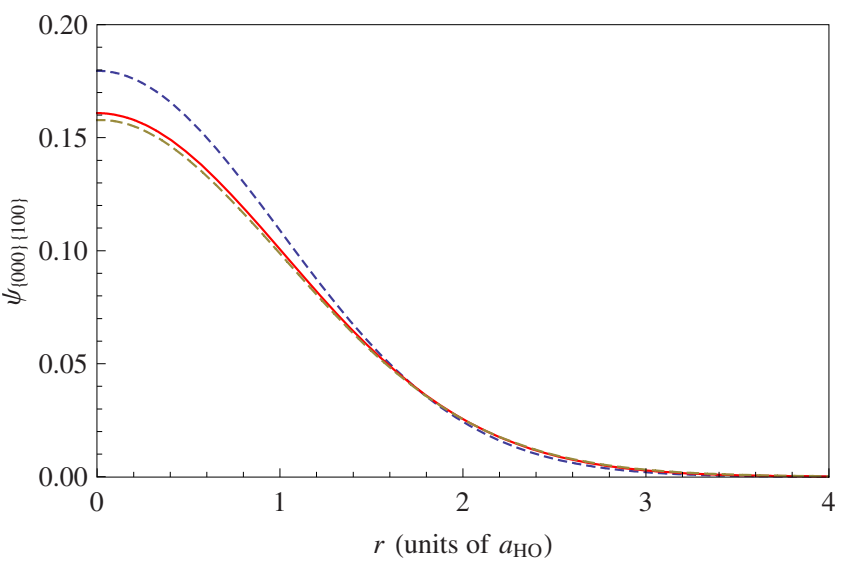

FIG. 3. (Color online) Comparison of the exact and perturbative wave functions in the lowest band, for $a=0$. Shown are the zeroth order $\mathrm{HO}$ solution (dashed line), the first-order corrected solution (solid line), and the exact localized Wannier function (dash-dotted line), for CM coordinate $R=0$. The relative coordinate is scaled on $a_{\mathrm{HO}}$, with $a_{\mathrm{HO}}=\sqrt{2 \hbar /(m \omega)}$ as the harmonic-oscillator length of relative motion.

\section{SPIN DYNAMICS}

In this section we apply our model to the spin-dynamics experiment, as carried out in the Bloch group [2,3]. This allows us to investigate systematically the effects of anharmonic terms and a nonzero interatomic interaction.

In an optical lattice one is able to trap several spin states at the same time. Starting with atoms prepared in a specific one-particle (hyperfine) spin state $\left|f, m_{f}\right\rangle$, collisions between two such atoms give access to other two-particle spin configurations. When only weak magnetic fields are applied, the total magnetization is conserved, and therefore coherent collisions between two-particle states of equal total two-particle spin $F$ occur. This can be described by a Rabi-type model. For atoms prepared in $f=1$, which is the case we will treat here, this is only a two level system, with effective Rabi frequency

$$
\Omega_{\mathrm{if}}^{\prime}=\sqrt{\Omega_{\mathrm{if}}^{2}+\delta_{\mathrm{if}}^{2}} .
$$

Here $\Omega_{\text {if }}$ is the bare Rabi frequency depending on the coupling strength of the spin-changing collision. Detuning $\delta_{\text {if }}$ contains two contributions,

$$
\delta_{\text {if }}=\delta_{0}+\delta\left(B^{2}\right)
$$

$\delta_{0}$ is given by the difference of two interaction energies corresponding to collisions that leave the spin configuration unchanged, and $\delta\left(B^{2}\right)$ is a second-order Zeeman shift between the initial and final states. By performing the experiment at different magnetic field strengths $B$, the Bloch group was able to subtract $\Omega_{\text {if }}$ and $\delta_{0}$, thereby being able to derive precise values for relative interaction strengths of rubidium. Treating the interaction energy as a linear perturbation in the parameter $k_{L} a$, one can derive the Rabi parameters as 
TABLE I. Theoretical predictions for the scattering lengths of the $F=0$ and $F=2$ channel, for atoms with one-particle spin $f=1$, based on accurate rubidium interaction potentials [4]. Here $F$ is the total two-particle spin. The values are given in units of the Bohr radius $a_{0}$.

\begin{tabular}{cc}
\hline \hline & $\begin{array}{c}a_{F} \\
\left(\text { units of } a_{0}\right)\end{array}$ \\
\hline 0 & $101.78 \pm 0.2$ \\
2 & $100.40 \pm 0.1$ \\
\hline \hline
\end{tabular}

$$
\Omega_{\mathrm{if}}=\frac{2 \sqrt{2}}{3 \hbar} \tilde{U}\left(a_{2}-a_{0}\right), \quad \delta_{0}=\frac{1}{3 \hbar} \tilde{U}\left(a_{2}-a_{0}\right) .
$$

The factor $\tilde{U}$ is here defined as

$$
\tilde{U}=\frac{4 \pi \hbar^{2}}{m} \int d^{3} x|\psi|^{4}
$$

with $\psi$ as the lowest HO eigenfunction. $\tilde{U}$ depends on the lattice depth but is independent of the scattering length. Hence in this approach, differences between interaction energies are due to scattering-length differences only. The values of the scattering lengths $a_{F}, F=0,2$, corresponding to collisions in subchannel $F$, are calculated based on highly accurate rubidium potentials [4], and are given in Table I. Note that $\psi$ can deviate significantly from the abovementioned solution of two interacting atoms in a trap, and therefore it can be expected that a proper treatment of interatomic interactions could have a large impact on the Rabi frequency. While this effect will be mostly pronounced when close to a Feshbach resonance, an effect can also be expected when highly accurate measurements are performed, as in the experiment of Widera et al.

The experimental results for the scattering lengths, based on the above Rabi model [2], agreed just within error bars with the predictions. This apparent discrepancy was most clearly seen for the $f=1$ case. We will now investigate this $f=1$ experiment by calculating the interaction strengths from our lattice model, and analyze the effects of anharmonic terms and exact interatomic interactions. Note that anharmonic corrections were also taken into account in Ref. [3], which already led to a better agreement between theory and experiment. We can most clearly compare theory and experiment by regarding the frequency $\Omega_{\text {if }}^{\prime H O}$ at $B=0$ since the field dependence does not depend on the interatomic interactions.

As a starting point for comparison, we apply the Rabi model as described above, with $\widetilde{U}$ calculated from HO solutions, and we use the scattering lengths from Table I to obtain the effective Rabi frequency. This gives $\Omega_{\text {if }}^{\prime \mathrm{HO}}(B=0)$ $=2 \pi \times 49.27 \mathrm{~Hz}$. Then, we first calculate $\Omega_{\text {if }}^{\prime}(B=0)$ by using Wannier functions in the expression for $\widetilde{U}$, in order to analyze the effects of anharmonic terms only. Wannier functions are exact solutions for a lattice without interactions. This is similar to the procedure performed by Widera et al. [3]. Second, we want to analyze the effect of having exact inter-
TABLE II. (Left column) Correction factor $\Omega_{\mathrm{if}}^{\prime} / \Omega_{\mathrm{if}}^{\prime \mathrm{HO}}$ of the effective Rabi frequency $\Omega_{\mathrm{if}}^{\prime}$, compared with the same quantity computed in the HO approximation with the interaction treated as linear perturbation. The first row shows the result when only anharmonic effects are taken into account. The result in the second row is obtained with only higher order interaction effects taken into account. In the third row the results are given for both effects acting together. Right column: corresponding values for the ratio between the theoretical $\Omega_{\text {if }}^{\prime}$ and the experimentally obtained effective Rabi frequency $\Omega_{\text {if }}^{\prime \exp }=2 \pi \times(35.4 \pm 0.7) \mathrm{Hz}$.

\begin{tabular}{lcc}
\hline \hline Included corrections & $\Omega_{\mathrm{if}}^{\prime} / \Omega_{\mathrm{if}}^{\prime \mathrm{HO}}$ & $\Omega_{\mathrm{if}}^{\prime} / \Omega_{\mathrm{if}}^{\prime \exp }$ \\
\hline Anharmonic & 0.897 & 1.25 \\
Interatomic interactions & 1.033 & 1.43 \\
Anharmonic +interatomic interactions & 0.935 & 1.30 \\
\hline \hline
\end{tabular}

atomic interactions only, and calculate the Rabi parameters by using the solutions for two interacting atoms in a harmonic trap;

$$
\begin{aligned}
\Omega_{\mathrm{if}} & =\frac{2 \sqrt{2}}{3 \hbar}\left[E_{\mathrm{int}}\left(a_{2}\right)-E_{\mathrm{int}}\left(a_{0}\right)\right], \\
\delta_{0} & =\frac{1}{3 \hbar}\left[E_{\mathrm{int}}\left(a_{2}\right)-E_{\mathrm{int}}\left(a_{0}\right)\right],
\end{aligned}
$$

where

$$
E_{\text {int }}(a)=E(a)-E(a=0) .
$$

Here we define the total energy $E(a)=E_{S s}^{(0)}(a)=E_{S}+e_{s}(a)$, with $S=\{0,0,0\}$ and $s=\{3,0,0\}$, according to the solution of two interacting atoms in a trap [14]. Third, the same is done but also with the anharmonic terms included by taking $E(a)=E_{S s}(a)$, in order to compute the combined effect. The results are shown in Table II by calculating the ratio of the different $\Omega_{\mathrm{if}}^{\prime}(B=0)$ over the initial frequency $\Omega_{\mathrm{if}}^{\prime \mathrm{HO}}(B=0)$. The table also shows a comparison with the experimentally obtained effective Rabi frequency. In all calculations a lattice depth of $V_{0}=45 E_{\text {rec }}$ is used. Note that the third calculation is the most precise one, with exact interactions, giving rise to modified wave functions and energy levels compared to the HO calculation. Moreover, the anharmonic effects are also included via perturbation theory up to high precision. An upper bound for the computational precision is $1 \%$, which is estimated by the contribution of second-order terms to $\Omega_{\text {if }}^{\prime}$. The contribution of tunneling is less than $2 \%$ of the secondorder correction at a lattice depth $V_{0}=45 E_{\text {rec }}$.

From the results we find that anharmonic terms induce a negative shift of order of $10 \%$. To the contrary, higher order interaction effects induce a positive shift, which is of order of $3 \%$. Hence, anharmonic corrections are dominant while the first-order approximation for the interaction energy is already fairly accurate. The net result is a $7 \%$ improvement with respect to the initial model of the Bloch group. Although the theoretical and experimental values still differ by $30 \%$, this is just within theoretical and experimental error bars. Note that the largest contribution to the theoretical error bar is due to the small difference $a_{2}-a_{0}$, which is only a 
percent of the values of $a_{0}$ and $a_{2}$ themselves. A second issue is a possible systematic error in $\widetilde{U}$, related to the uncertainty in the lattice depth. At present there is no direct measurement of this coupling constant.

\section{ADIABATIC CREATION OF FESHBACH MOLECULES WITH A CM MOTION}

In the previous section we applied our model to nonresonantly interacting atoms in the lowest lattice band. This means that the total energy is relatively close to the noninteracting ground states when compared to the level splitting between ground-state and nonground-state levels. We take the coupling between $\mathrm{CM}$ and relative motions into account but we neglect the interaction induced coupling between levels since the energy distance to nonground-state levels is too large. For example, with the lattice depth and scattering lengths used in the previous section, we have $E^{(1)}$ $\simeq 0.23 \hbar \omega / 2$ while the largest coupling is obtained from the overlap with the next spherical symmetric level:

$$
\frac{\left|\left\langle\psi_{\{000\}\{300\}}^{(0)}\left|H^{\prime}\right| \psi_{\{000\}\{500\}}^{(0)}\right\rangle\right|^{2}}{E_{\{000\}\{300\}}^{(0)}-E_{\{000\}\{500\}}^{(0)}} \leq 0.008 \hbar \omega / 2 .
$$

However, when the interaction strength is increased, several $\mathrm{CM}$ and relative states become (nearly) degenerate, and therefore first-order lattice perturbation terms will already result in an efficient coupling. In this section, we will show that the coupling between $\mathrm{CM}$ and relative states implies that atoms can be transferred into molecules, with a simultaneous transfer of quantized energy of the relative motion to the $\mathrm{CM}$ motion, and vice versa. Here molecules should be thought of as two atoms bound together via the interatomic potential, cf. Eq. (10).

Tuning the scattering length through resonance can give rise to a transfer of atoms from one $\mathrm{HO}$ level to the next one [12], and under adiabatic conditions this can be observed experimentally [15]. In Fig. 1 this is illustrated for the relative energy. A similar graph for the total energy is shown in Fig. 4, with thin gray lines for the lowest HO levels including interaction. From this plot we find that degeneracies occur around resonance $(1 / a \sim 0)$, and for the limit $a \rightarrow 0^{-}$. Anharmonic terms can lift these degeneracies, and couple the $\mathrm{CM}$ and relative levels. Within first-order perturbation theory, states of equal total quantum number $S_{1}+S_{2}+S_{3}+n$ are coupled, with coupling strengths dependent on both scattering length and lattice depth. To compute the first-order corrected energy we diagonalize the matrix whose elements are given by

$$
\left\langle\psi_{S S}^{(0)}\left|H_{\mathrm{HO}}+H^{\prime}\right| \psi_{S^{\prime} s^{\prime}}^{(0)}\right\rangle=\delta_{S S^{\prime}} \delta_{S s^{\prime}} E_{S s}^{(0)}+\left\langle\psi_{S S}^{(0)}\left|H^{\prime}\right| \psi_{S^{\prime} s^{\prime}}^{(0)}\right\rangle .
$$

Here $\delta_{i i^{\prime}}$ is the Kronecker delta. In the limit $a \rightarrow 0^{-}$these states are degenerate and correspond to a nonground state of the lattice well (top right of Fig. 4). For $a$ being small and positive the interaction induced coupling becomes negligible, as was the case in the previous section.

Several coupling terms are zero due to the symmetry of the perturbation term. For the actual computation of the cou-

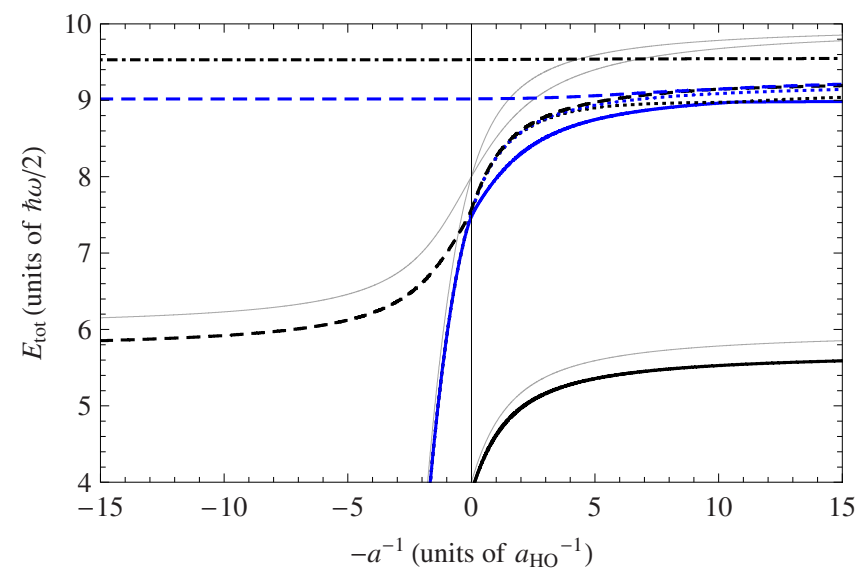

FIG. 4. (Color online) Total energy of the combined relative and $\mathrm{CM}$ system, as a function of the reciprocal scattering length. The upper thick lines represent the six states that are coupled by anharmonic terms. Thin gray lines denote the zeroth order HO levels. The scattering length is scaled on $a_{\mathrm{HO}}$, with $a_{\mathrm{HO}}=\sqrt{2 \hbar /(m \omega)}$ as the harmonic-oscillator length of the relative motion.

pling terms, we can integrate the three different components of the CM motion separately:

$$
\begin{aligned}
\left\langle\psi_{S s}^{(0)}\left|H^{\prime}\right| \psi_{S^{\prime} s^{\prime}}^{(0)}\right\rangle= & \left\langle\phi_{s}\left|\left\langle\Phi_{S_{1}}\left|H_{j}^{\prime}\right| \Phi_{S_{1}^{\prime}}\right\rangle\right| \phi_{s^{\prime}}\right\rangle \delta_{S_{2} S_{2}^{\prime}} \delta_{S_{3} S_{3}^{\prime}} \\
& + \text { (cyclic permutations) } .
\end{aligned}
$$

The function

$$
H_{j}^{\prime}=V_{j}\left(R_{j}+\frac{r_{j}}{2}\right)+V_{j}\left(R_{j}-\frac{r_{j}}{2}\right)-\frac{1}{2} m \omega^{2}\left(2 R_{j}^{2}+\frac{1}{2} r_{j}^{2}\right)
$$

is even in $R_{j}$ and $r_{j}$. Hence, CM states with $S_{j}+S_{j}^{\prime}$ do not couple, i.e., there is only coupling between $\mathrm{CM}$ states with equal parity. Also, due to the cubic symmetry of the perturbation term, the integration has to be performed only for one axis for each combination $S_{j}$ and $S_{j}^{\prime}$. The parity of the relative $\mathrm{HO}$ states is determined by the angular part since the radial part is spherically symmetric. For spherical harmonics $Y_{l}^{m}(\theta, \phi)$ the parity is given by

$$
Y_{l}^{m}(\pi-\theta, \phi+\pi)=(-1)^{l} Y_{l}^{m}(\theta, \phi) .
$$

Consequently, all states with $l+l^{\prime}$ odd vanish. Also with $l$ $+l^{\prime}$ even, not all combinations $m$ and $m^{\prime}$ give rise to coupling. In particular, there are vanishing contributions for odd values of $\left|m-m^{\prime}\right|$ since these introduce an odd term,

$$
e^{-i\left(m-m^{\prime}\right) \phi} \propto(x-i y)^{m-m^{\prime}},
$$

while the other contributions are even.

We now restrict ourselves to the coupling between the lowest symmetric states $\left|\psi_{S s}\right\rangle$ that are perturbed by the cubically symmetric perturbation term. These states can be divided in two sets. The first set consists of a $\mathrm{CM}$ ground state and excited relative states with labels $S=\{0,0,0\}$ and $s$ $=\{3, l, m\}$, and the second set consist of excited CM states and a relative ground state with $S=\{2,0,0\}$ (and cyclic permutations), and $s=\{1,0,0\}$, which contains a molecular 


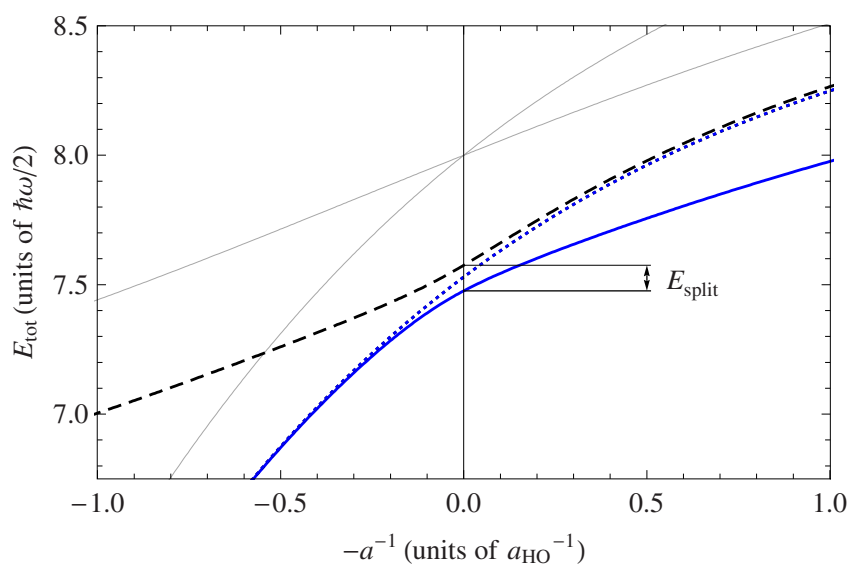

FIG. 5. (Color online) Zoom in of Fig. 4 around resonance $(1 / a=0)$. The scattering state (dashed line) is coupled to one excited CM state (solid line), which contains a molecular bound state for positive $a$, while the other two states (dotted line) are degenerate and remain uncoupled around resonance. The splitting energy is indicated by $E_{\text {split }}$.

bound state for positive $a$. In the first case, quantum numbers can be $l, m=\{0,0\},\{2,0\},\{2,-2\},\{2,+2\}$; hence four relative states in total. The number of coupled relative states exceeds the number of coupled CM states since the spherical harmonics functions have some redundancy for expressing cubic symmetry. However, the actual number of relative states that are coupled reduces to three when we take proper linear combinations of $d$ waves. Hence, dictated by the symmetry of the anharmonic terms, six states are coupled in total. Note that it is quite remarkable that a coupling to relative $d$-wave motion is possible without an interatomic coupling on short range. This coupling between $s$ and $d$ waves is indirect. The $s$ waves are coupled to excited CM states which in turn are coupled to $d$ waves, illustrating the long-range character of the anharmonic coupling.

The main result of this section is shown in Fig. 4. Total energy for the combined relative and CM motions is shown as a function of the scattering length. Thick lines represent the six states that are coupled by anharmonic terms. Thin gray lines indicate the corresponding zeroth order HO levels. Also the perturbed uncoupled ground state is shown using a thick solid line. The calculation is done for a lattice depth of $V_{0}=25 E_{\text {rec }}$. Figure 4 shows the presence of $d$-wave states in the upper two solutions, indicated with dashed-dotted and dashed lines. These solutions have only weak dependence on the $s$-wave scattering length, owing to the indirect coupling via the excited CM states. Below the $d$-wave states a dashed solution is shown, which connects asymptotically to two consecutive levels, similar to the results of the Busch model for a $s$-wave scattering state. However, the three remaining solutions (dotted, dotted, and solid) are of a different nature. These reflect the presence of excited CM states, and asymptotically connect to the molecular state of the relative motion for positive $a$. A zoom in around resonance $(1 / a=0)$ is given in Fig. 5. It can be clearly seen that the coupling between relative and CM motions, caused by the long-range anharmonic terms, gives rise to a transfer of energy between these two motions. On resonance only the dashed and solid lines are coupled, giving rise to an energy splitting indicated by $E_{\text {split }}$. The two dotted solutions are coupled at small negative values of the scattering length and with much smaller splitting energies.

It is interesting to consider possible applications of this anharmonic coupling at long range, and interatomic coupling at short range. This can be done by exploiting different time scales when changing the scattering length. The scattering length can be changed by utilizing the magnetic field dependence of the scattering length via a Feshbach resonance.

One can transfer for instance atoms from the lowest band into the next band by ramping the magnetic field, and by slowly ramping back associate molecules with an excited CM motion. This would result in molecular energy levels that deviate significantly from the energy of ground-state molecules labeled with $S=\{0,0,0\}$ and $s=\{1,0,0\}$. Such higher molecular levels correspond to molecules in different (partly filled) Brillouin zones, which should be possible to detect [16]. We note that these excited CM molecules, compared to ground-state molecules, have a larger spatial extent in the CM motion.

The typical time scale $\tau$ for slowly ramping back is given by $\tau \gg \hbar / E_{\text {split }}$. In contrast to other lattice induced molecules, see, e.g., [17-19], these excited CM molecules do not arise from tunneling to neighboring sites but from the anharmonic shape of a single lattice site. In addition, these excited CM molecules could be observable even for rather deep lattices $\left(V_{0}>50 E_{R}\right)$ since the anharmonic effects decay only weakly with increasing lattice depth, whereas tunneling effects decay exponentially.

\section{CONCLUSION}

In conclusion, we proposed an easy to use method to solve interacting atoms in optical lattices, where the relative and center-of-mass motions of the two interacting atoms are coupled via the anharmonic terms of the lattice. The interactions are treated exactly using a boundary condition rising from a pseudopotential. The anharmonic terms of the lattice potential are treated as a perturbation of the exact solution for two cold interacting atoms in a harmonic trap. We applied this model to the Mainz spin-dynamics experiment [2,3] for $f=1$. The interaction energy is computed as the difference between two-atom energy levels with and without interactions. This model gives a more rigorous interpretation of the experiment compared to previous descriptions in terms of two-body scattering properties. We find that the derived scattering lengths agree within the experimental and theoretical error bars. Apart from applying our model to spin dynamics, we are in a good position to analyze future optical lattice experiments where the interactions are made very strong by utilizing Feshbach resonances. Strong interactions can induce coupling between the relative and center-of-mass motions, which allows for an energy exchange between these two motions, and which can be used to produce (Feshbach) molecules with an excited center-of-mass motion. This model can also be used as a starting point for a description of photoassociation in an optical lattice near a Feshbach resonance $[20,21]$. 


\section{ACKNOWLEDGMENT}

This work was supported by the Netherlands Organization for Scientific Research (NWO).

\section{APPENDIX: EXACT LATTICE SOLUTION WITHOUT INTERACTION}

In this appendix we derive exact solutions for noninteracting atoms in a cubic optical lattice, which we need to estimate the effect of tunneling and anharmonic terms in our system of consideration. First we will introduce Bloch functions and Wannier functions for the lattice. Second we will show how these functions can be expressed in terms of Mathieu functions.

For noninteracting particles there is separation in the single-particle coordinates, and the 3D wave function for each particle can be conveniently written as product $1 \mathrm{D}$ wave functions. We thus have to solve a 1D Schrödinger with a periodic potential. According to the Bloch theorem, the solution can be written as

$$
\psi_{q s}(x)=e^{i q x} u_{q s}(x)
$$

with quasimomentum $q$, band index $s$, and $u_{q s}(x)$ having the same periodicity as the potential. This Bloch function can also be expressed as a sum over localized solutions. This follows when we consider the Bloch function as function of $q$. Because $\psi_{q s}$ is periodic in $q$, we can write it as a Fourier series in $q$,

$$
\psi_{q s}(x)=\left(\frac{d}{2 \pi}\right)^{1 / 2} \sum_{n=-\infty}^{\infty} w_{s}(x-d n) e^{i q d n}
$$

with $d$ as the lattice spacing. The coefficients $w_{s}(x-d n)$ of this Fourier series are (regarded as function of $x$ ) the Wannier functions of band $s$. They are given explicitly in terms of Bloch functions by the inversion formula,

$$
w_{s}(x-d n)=\left(\frac{d}{2 \pi}\right)^{1 / 2} \int_{-\pi / d}^{\pi / d} e^{-i q d n} \psi_{q s}(x) d q .
$$

Hence, the Wannier function of a given band is a linear combination of all Bloch functions of that same band. An extensive analysis of the properties of Wannier functions is given by [22]. For example, it can be proven that, with Bloch functions normalized over a single site, such that

$$
\frac{2 \pi}{d} \int_{0}^{d}\left|\psi_{q s}(x)\right|^{2} d x=1
$$

Wannier functions at different sites and different bands are orthonormal

$$
\int_{-\infty}^{\infty} w_{s^{\prime}}^{*}\left(x-d n^{\prime}\right) w_{s}(x-d n) d x=\delta_{s^{\prime} s} \delta_{n^{\prime} n},
$$

with $\delta_{m m^{\prime}}$ as the Kronecker delta. Although the Wannier functions are a linear combination of Bloch functions, they do not satisfy the time-independent Schrödinger equation. This is because Bloch functions of different quasimomentum have different energies. In addition, Wannier functions do not satisfy periodic boundary conditions; however, by construction they are required to decay exponentially for large $\tilde{x}$. To obtain the differential equation of the Wannier function, we should similarly expand the energy in $q: E_{q}=\Sigma_{n} e_{n} e^{i q d n}$. In case the lattice is very deep and hence the bands are practically flat, i.e., $d E / d q=0$, the differential equation for the Wannier function does reduce to the Schrödinger equation.

For the case of an optical lattice potential of the form Eq. (4), Bloch and Wannier functions can be obtained in closed form, including the corresponding characteristic energies (band structure). This can be seen with the introduction of the parameters

$$
e=\widetilde{E}-\frac{1}{2} \widetilde{V}_{0}
$$

$$
h=-\frac{1}{4} \tilde{V}_{0},
$$

in the 1D Schrödinger equation. This gives

$$
\frac{d^{2} \psi}{d \tilde{x}^{2}}+(e-2 h \cos 2 \widetilde{x}) \psi=0
$$

where $\tilde{x}=k_{L} x, \quad \tilde{E}=E / E_{\mathrm{rec}}$, and $\tilde{V}_{0}=V_{0} / E_{\mathrm{rec}}$, with $E_{\mathrm{rec}}$ $=\hbar^{2} k_{L}^{2} /(2 m)$ as the recoil energy and $k_{L}$ as the wave number corresponding to the laser frequency. The standard solutions of Eq. (A8) are called Mathieu functions, denoted as $c e_{\nu}(\tilde{x}, h)$ and $s e_{\nu}(\tilde{x}, h)$, which are even and odd as function of $\tilde{x}$, respectively. Mathieu functions and their characteristic energies are conveniently implemented in modern computer algebra systems. Here we will mention only briefly the properties which are relevant for us; for an extensive discussion of Mathieu functions we refer to $[13,23]$.

For noninteger $\nu$, the functions $c e_{\nu}$ and $s e_{\nu}$ are two independent solutions with characteristic energy $e_{\nu}(h)$. At a given lattice depth $h, e_{\nu}(h)$ as function of $\nu$ gives the band structure in the extended zone scheme ( $\nu$ plays the role of quasimomentum). With $\nu=n$ integer valued, $c e_{n}$ and $s e_{n}$ are periodic with the same periodicity as the lattice but at different energies: $e_{n}^{c}(h)$ and $e_{n}^{s}(h)$. The other independent solutions for this case contain a logarithmic term that does not satisfy periodic boundary conditions. The characteristic energies $e_{n}^{c}(h)$ and $e_{n}^{s}(h)$ correspond to band tops and band bottoms. For our optical lattice potential we have $h<0$. In this case the band top and bottom of even bands ( $s$ even) are $e_{2 n-1}^{c}(h)$ and $e_{2 n-2}^{c}(h)$, respectively. The band top and bottom of the odd bands ( $s$ odd) are given by $e_{2 n}^{s}(h)$ and $e_{2 n-1}^{s}(h)$. Hence we have $e_{0}^{c}<e_{1}^{c}<e_{1}^{s}<e_{2}^{s}<e_{2}^{c}<\ldots$. These band tops and bottoms are used in Sec. II to compute $E_{t}$ and $E_{b}$, and estimate the effects of tunneling and anharmonic terms. 
With the general solution of the Schrödinger equation in closed form, the Bloch function can be obtained by imposing boundary conditions according to the Bloch theorem. With this Bloch function, we also have the Wannier function in closed form, as an integral over Mathieu functions, weighted by an exponential. The Wannier function obtained this way is used in Sec. II to check the accuracy of our model for the case without interaction.
[1] K. Winkler, F. Lang, G. Thalhammer, P. v. d. Straten, R. Grimm, and J. H. Denschlag, Phys. Rev. Lett. 98, 043201 (2007).

[2] A. Widera, F. Gerbier, S. Fölling, T. Gericke, O. Mandel, and I. Bloch, Phys. Rev. Lett. 95, 190405 (2005).

[3] A. Widera, F. Gerbier, S. Fölling, T. Gericke, O. Mandel, and I. Bloch, New J. Phys. 8, 152 (2006).

[4] E. G. M. van Kempen, S. J. J. M. F. Kokkelmans, D. J. Heinzen, and B. J. Verhaar, Phys. Rev. Lett. 88, 093201 (2002).

[5] J. F. Bertelsen and K. Mølmer, Phys. Rev. A 76, 043615 (2007).

[6] F. Deuretzbacher, K. Plassmeier, D. Pfannkuche, F. Werner, C. Ospelkaus, S. Ospelkaus, K. Sengstock, and K. Bongs, Phys. Rev. A 77, 032726 (2008).

[7] K. Huang and C. N. Yang, Phys. Rev. 105, 767 (1957).

[8] The scattering length can be replaced by a function of the relative momentum in order to get an advanced energy dependence of the scattering phase shift. In case of a constant $a$, one accounts for a trivial energy dependence $\delta(k)=\arctan k a$, which is valid for broad Feshbach resonances and nonresonant scattering.

[9] For the validity of this approximation it is important that the variation in the lattice potential is negligible over the range of the real interatomic potential. For more discussion on the validity of the pseudopotential, see, e.g., Ref. [24].

[10] G. F. Gribakin and V. V. Flambaum, Phys. Rev. A 48, 546 (1993).

[11] N. W. Ashcroft and N. D. Mermin, Solid State Physics (Saunders College, London, 1976).
[12] T. Busch, B. G. Englert, K. Rzazewski, and M. Wilkens, Found. Phys. 28, 549 (1998).

[13] P. M. Morse and H. Feshbach, Methods of Theoretical Physics (McGraw-Hill, New York, 1953).

[14] The lowest state at positive scattering length is the molecular bound state. Because we aim to describe scattering between atoms here, we use the one-but-lowest symmetric state, with $s=\{3,0,0\}$.

[15] T. Stöferle, H. Moritz, K. Günter, M. Köhl, and T. Esslinger, Proceedings of the XVII International Conference Laser Spectroscopy (World Scientific, Singapore, 2006), p. 283.

[16] M. Köhl, H. Moritz, T. Stöferle, K. Günter, and T. Esslinger, Phys. Rev. Lett. 94, 080403 (2005).

[17] P. O. Fedichev, M. J. Bijlsma, and P. Zoller, Phys. Rev. Lett. 92, 080401 (2004).

[18] M. Wouters and G. Orso, Phys. Rev. A 73, 012707 (2006).

[19] K. Winkler, G. Thalhammer, F. Lang, R. Grimm, J. Hecker Denschlag, A. J. Daley, A. Kantian, H. P. Büchler, and P. Zoller, Nature (London) 441, 853 (2006).

[20] D. Jaksch, V. Venturi, J. I. Cirac, C. J. Williams, and P. Zoller, Phys. Rev. Lett. 89, 040402 (2002).

[21] M. Junker, D. Dries, C. Welford, J. Hitchcock, Y. P. Chen, and R. G. Hulet, Phys. Rev. Lett. 101, 060406 (2008).

[22] W. Kohn, Phys. Rev. 115, 809 (1959).

[23] N. W. McLachlan, Theory and Application of Mathieu Functions (Oxford University Press, London, 1951).

[24] R. Stock, I. H. Deutsch, and E. L. Bolda, Phys. Rev. Lett. 91, 183201 (2003). 